\section{Shared decision-making in the chronic care of Primary Immunodeficiency}

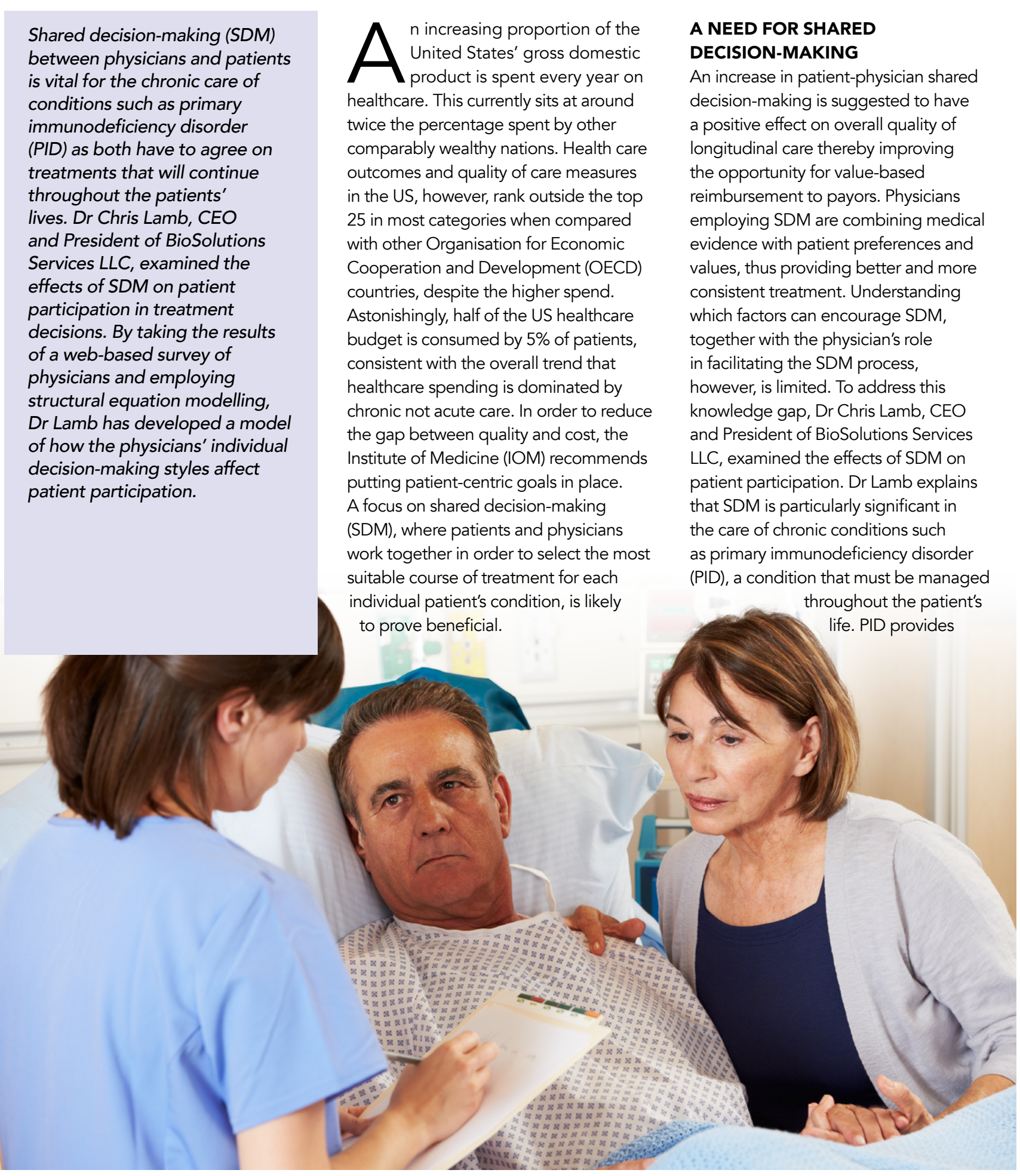

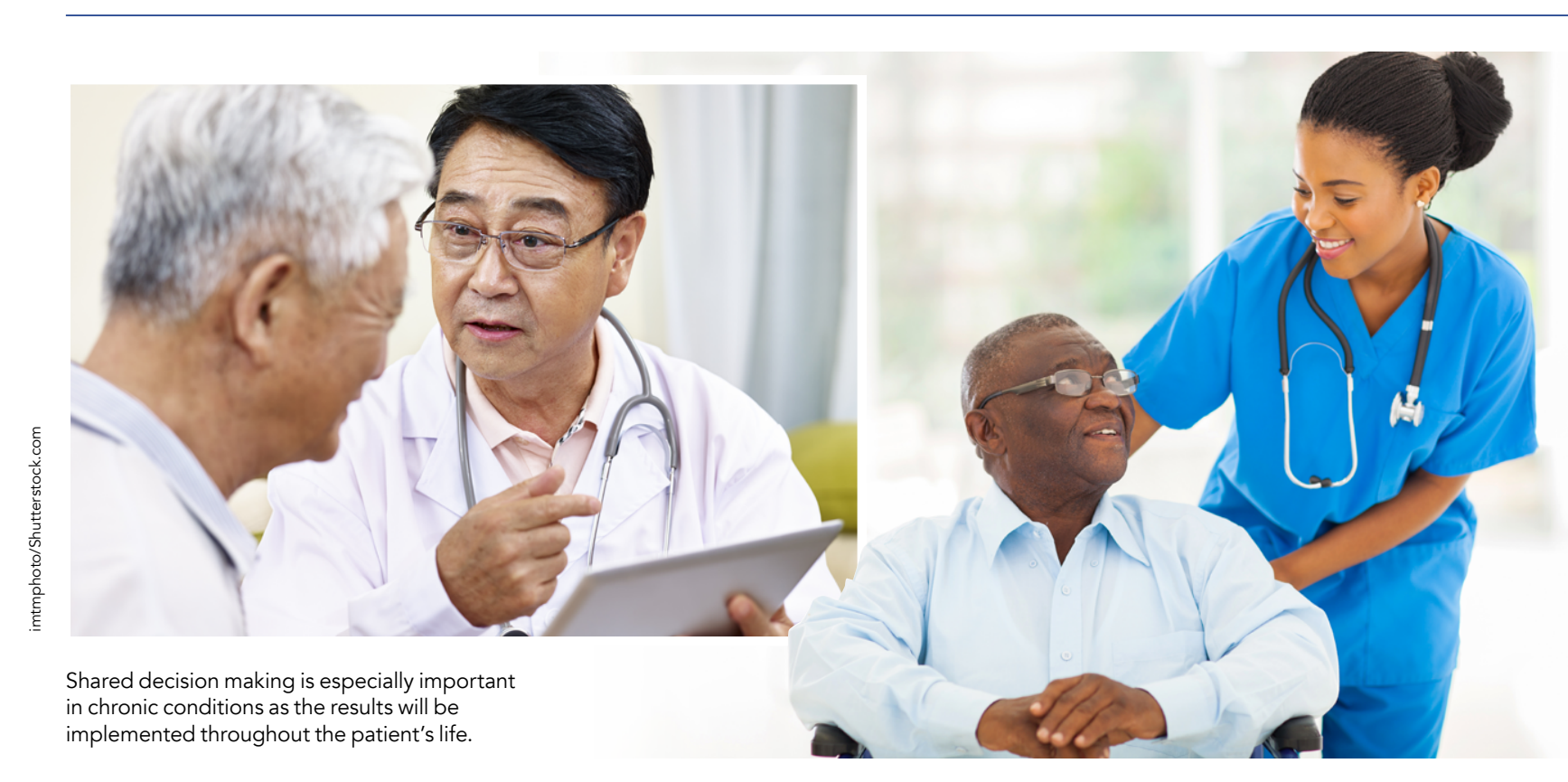

an excellent vehicle to study SDM given its challenging dimensions and It is expected that the findings from this study will be applicable to many other chronic diseases.

PRIMARY IMMUNODEFICIENCY DISORDER (PID)

Primary immunodeficiency disorder includes more than 350 rare, chronic disorders that are the result of part of the body's immune system not functioning properly, or not functioning at all. These conditions are usually caused by hereditary or genetic defects. They can affect anyone, regardless of age or gender, but are not contagious. People to ining from PD are more susceptible helth problic and the dever in of serious and debilitating illesses PID is difficult to diagnose and this, together with the cost of drugs and preventable hospitalisation, makes PID one of the most expensive diseases to treat. SDM is of particular significance to chronic conditions such as PID, as patients and physicians have to agree on treatments that will continue throughout the patients' lives.

\section{DUAL PROCESS THEORY} Concepts available to the medical decision-makng process include the dual process theory of reasoning. Dual process theory assimilates two forms of thinking. The first (system 1) involves our intuition, or gut-feeling, in our subconscious. This heuristic g relies on experience. more deliberate; it requires effort to consciously work through different

education, and race influence the effect of their decision-making style on SDM? questionnaire, which was grounded on lished validated scales. This meant

Approximately 17,500 patients would have received treatment from the physicians in the sample group.

considerations, apply various concepts and models and weigh them all up. Physicians will employ either system depending on circumstances. It has been shown, however, that individ tend to have a preference for one been observed to often ime also both rational and heuristic methods, frequently one after the other.

\section{STUDY DESIGN}

ate the relationships between physician decision-making styles and other physician characteristics relating to SDM, Dr Lamb and his colleagues posed the following research questions and constructed the associated hypotheses

A. Do physician decision-making styles affect patient participation in SDM as mediated by patient-centric car B. Does the level of trust between physicians and patients influence the
effect of physician decision-making sty/ en SDM?
onsictian decision-making style

C. Do physician traits such as age, gender that structural equation modelling could be employed to measure how these characteristics were interrelated.

MEASURING PHYSICIANS DECISION-MAKING STYLE AND PATIENT-CENTRIC APPROACH Having previously carried out a smaller quaantitative study and sought physician treating PID with immunoglobhysicic participate. A web-based survey was completed by 330 physicians practising of 60 questions designed to elicit the physicians' decision-making style, their patient-centric approach, and measure their trust in patients. Physicians scored each question on a five-point Likert-type "strongly agrongly disagree" to $5=$

.

The physician's decision-making process in the US. The survey was made up from two variables: rational decisiondecision-makstem 2) is rational

The researchers then designed a survey scale with possible responses ranging was determined as the independent 


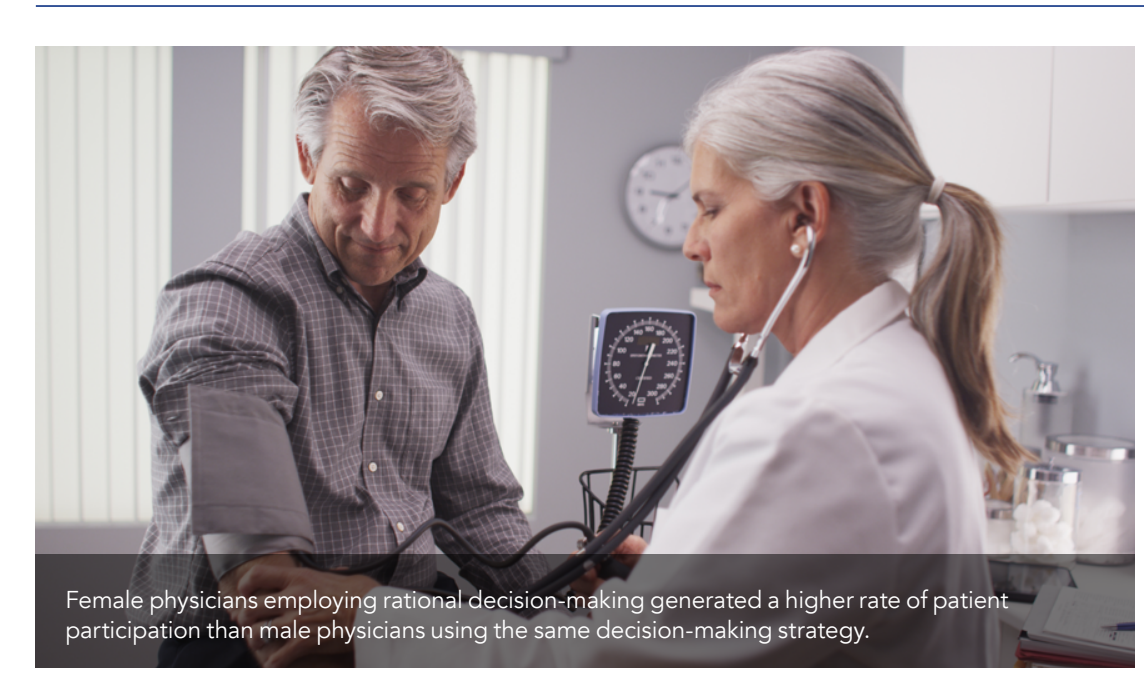

making and heuristic decision-making These were both analysed to meas each individual's affinity for either
analytical (rational) or empirical (heuristic analytical (rational)

Physicians' patient-centric approach was measured using the Patient-Practition trust in their patients S). The physicians information was gauged by means of Thom's 2011 Physician Trust in Patient include physicians' age and analysis was carried out using SPSS

\section{RESULTS}

Dr Lamb estimates that the 330 physicians $55 \%$ of all US patients with PID receiving treatment with immunoglobulin therapy Approximately 17,500 patients would have received treatment from the physicians in the sample group. Of the participating

The results suggested that there were differences relating to race, age, education, and gender.

Scale. The study assessed two aspects of patient participation in decision-making. Firstly, the patients' participation in the selection of their reatment schedules and administration methods, i.e. their was measured Secondly, patients' participation in choosing their medicaton or device, i.e. their participation in treatment tools, was measured using the Responsiveness to Patient Requests scale.

\section{STATISTICAL ANALYSIS}

Exploratory factor analysis and

confirmatory factor analysis, both standard multivariate statistical techniques, were employed to analyse the data and create a model of how physicians 'individual decision-making styles affect their patient decisions regarding their participation in treatment. Preliminary analysis suggested that a physician's age could have an effect as the older, more experienced immunologists had a discenbly dfferent

physicians, $74 \%$ were male (224), $72 \%$ were White (238) and 22\% specialise in allergy/ subsamples do for overlap.

The analysis revealed a statistically significant relationship between rational difference test was carried out to establis whether physician characteristics affect the level of patient participation. The results suggested that there were differences relating to race, age, education, and gender. There were no observed differences, however, relating to the physicians' trust in their patients to provide accurate information.

Dr Lamb's results suggest that in the case of older physicians (>50 years age), their rational decision-making increases patient participation with treatment protocols more than younger physicians ( $<50$ years age). Also, immunologists impleme participation with treatment protocols

Results also showed that physicins with PhDs encourage patient participation and therefore SDM, whether they employ a patient-centric approach or not. In addition, as the PhD-degreed physicians' age increases, so does their patients' participation rate when choosing treatment protocols.

Findings also revealed that female physicians employing rational decisionmaking generated a higher rate of patien participation than male physicians using the same decision-making strategy

\section{DIsCussion}

Dr Lamb and his colleagues have take the results of their web-based survey equation modelling to develop a model of how the physicians' individu decision-making styles affect patient participation in treatment decisions. The model also shows how a patient-centric approach to treatment can influence patient participation. Trust did not moderate the effect of decision-making on participation and was found to be an assumed component of the physicianpatient relationship.

Dr Lamb's research demonstrates how the different decision-making styles employed by physicians can affect patien's partipation in their treatment decision-making. Rational decisionparticipation with protocols while heuristic decision-making has a positive effect on participation with treatment tools. This study has also shown that patient participation is facilitated by a patientcentric approach to health care. Employing this approach can increase the likelihood of patient participation regardless of the physician's decision-making style.

Although this study concentrated on PID, further studies extending Dr Lamb's research to other chronic diseases are warranted. Dr Lamb recommends that Government policymakers, health care providers, patient organizations, and innovative drug companies should consider the type of interions and circumstances in which physician decision-

\section{Behind the Research}

\section{Dr Chris Lamb}

E: ccl48@case.edu T: $+13394406061 \quad$ W: https://www.linkedin.com/in/chris-lamb-phd-5b75601b/
W: https://www.pptaglobal.org/ W: Wtpp://www.info4pi.org/ W: $\mathbf{h t t p s : / / p r i m a r y i m m u n e . o r g / ~}$

\section{Research Objectives}

Dr Lamb's work examines the effects of shared decisionmaking on patient participation.

\section{Detail}

92 Irving Avenue

Englewood Cliffs

USA

Bio

Dr Lamb is an experienced Senior Healthcare Executive transitioning to academia. He has over 30 years operating in a dynamic, international competitive business environment with responsibility for: complex transactions, business development, R\&D, operations, sales \& marketing His research interests include: Decision Theory, Healthcare Economics, Entrepreneurship, and Organizational Design.

Collaborators

Yunmei Wang, BS, PhD, MBA (co-author)

Kalle Lyytinen, PhD (co-author)

Ryan Dagenais (coding, analysis,

CASEWESTERNRESERVE think beyond the possible'

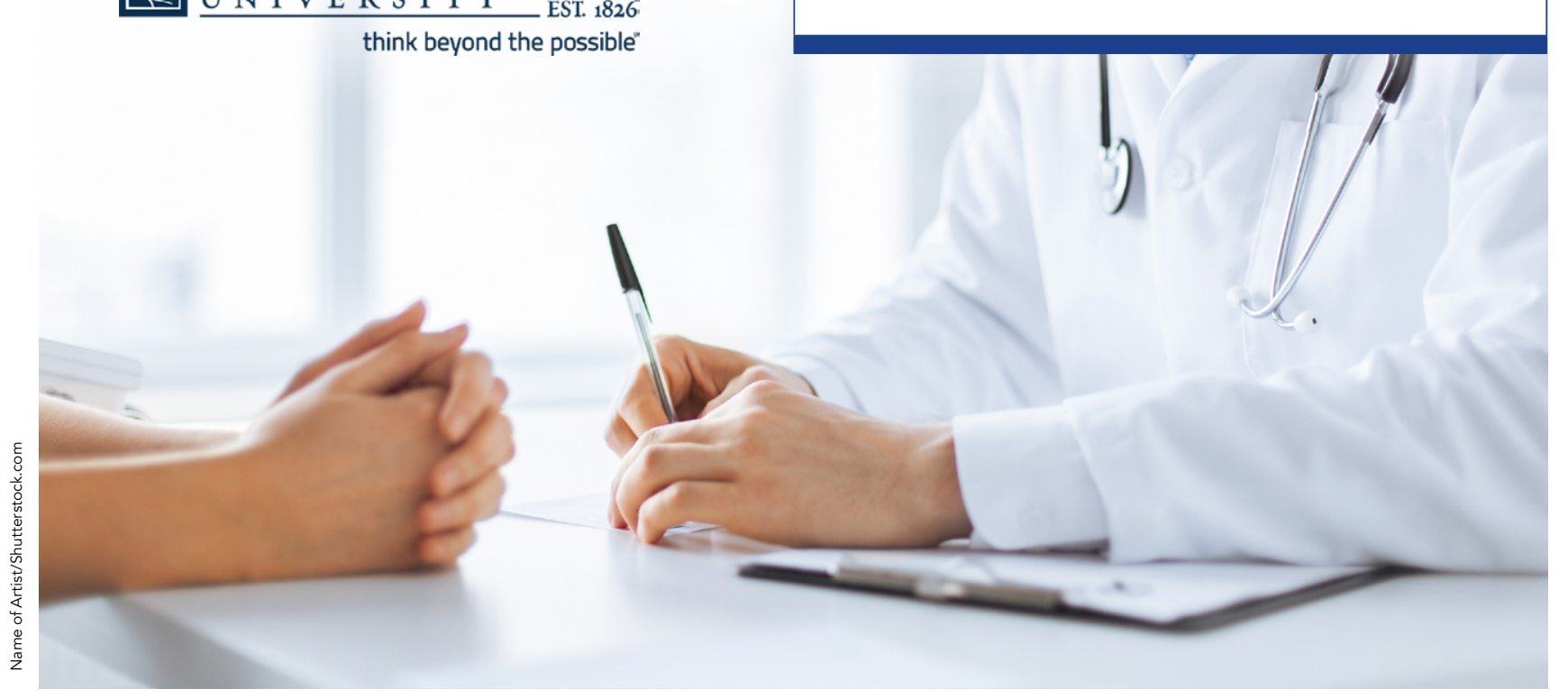

\section{References}

Lamb, C.C., Wang, Y., Lyytinen, K. (2019). Shared decision making: Does a physician's decision-making style affect patient participation in treatment choices for primary
immunodeficiency? Journal of Evaluation in Clinical Practice, [online] 1 - 9. Available at: https://doi.org/10.1111 jep.13162. [Accessed 3rd September 2019] physician decision-making in the treatment of haemophilia, org/10.1111/hae.13766. [Accessed 3rd September 2019]

Lamb, C. (2018). Physician-Patient Shared Decision Making in the Treatment of Primary Immunodeficiency: An InterviewBased Survey of Immunologists. LymphoSign Journal, lymphosign-2018-0010. [Accessed 3rd September 2019]

\section{Personal Response}

What initially prompted you to examine the effects of Making on patient participation?

II Having worked with different chronic diseases and thei associated patient advocacy groups over the past 30 years,
I though it was time to better understand how the physicianpatient interaction could be optimised. After a thorough literature review, it was clear that little research has been done to understand the physician perspective on SDM.
Lamb, C.C., Wolfberg, A., Lyytinen, K. (2019). UK vs US My work sought to address this research gap. 